\title{
Experimental spectrum of the cosmic ray variations within rigidity range of 1-20 GV in the Earth's orbit by the data of AMS-02
}

\author{
Victor G. Yanke ${ }^{\circledR}$, Anatoly V. Belov ${ }^{\circledR}$, Liudmila Trefilova, Pavel G. Kobelev ${ }^{\circledR}$, \\ Eugenia A. Eroshenko ${ }^{\circledR}$, Raisa Gushchina ${ }^{\circledR}$ \\ Correspondence \\ Pushkov Institute of Terrestrial Magnetism, Ionosphere and Radio Wave Propagation (IZMIRAN), Moscow, Russia, \\ yanke@izmiran.ru, abelov@izmiran.ru, trefilova@izmiran.ru,kosmos061986@yandex.ru, erosh@izmiran.ru, \\ rgus@izmiran.ru
}

\section{OPEN ACCESS}

This work is published under the Creative Commons Attribution 4.0 International license (CC BY 4.0) Please note that individual, appropriately marked parts of the work may be excluded from the license mentioned or may be subject to mer If such third paty mans. usuch third party material is not under the Creative Commons license any copying, editing or public reproduction is only permitted with the prior consent of the respective copy right owner or on the basis of relevan legal authorization regulations.

\section{Keywords}

long-term variations; AMS-02; spectrum of variations; ground-based detectors

\begin{abstract}
For the first time, based on the experimental data of AMS-02, a three-parameter spectrum of variations of galactic cosmic rays was obtained in the range of rigidity 1-20 GV, to which neutron monitors are most sensitive. It was found that during the period of negative polarity of the solar magnetic field, a power-law spectrum of variations is observed with a strong exponential decay in the region of high rigidity. When the polarity changes to positive at the beginning of the new 24 th solar cycle, the spectrum of cosmic ray variations becomes purely power-law. The transition to the experimentally obtained spectrum of variations will make it possible to remove a number of uncertainties and increase the accuracy of the analysis of data from the ground network of detectors. This will make it possible to retrospectively obtain fluxes of galactic protons with an average monthly resolution for the period of the space era based on ground-based monitoring.
\end{abstract}

\section{Introduction}

Despite the significant progress achieved in recent decades, the physical mechanisms underlying the modulation of galactic cosmic rays in the heliosphere still the subject of discussion. The complex process of particle transfer creates problems for the development of a universal picture of longterm variations in galactic cosmic rays, which covers a long observational period (on the order of 70 years) of continuous monitoring of cosmic radiation.

Experimental data on flux variations over the period from the mid-1950s to the present could be obtained with good accuracy only from continuous ground-based measurements on a network of cosmic ray detectors. The need to analyze the results of such long-term ground monitoring led to the creation of special methods. One of the earliest and most successful implementations of the global method was created in Yakutsk (Krymsky 1966, 1967, 1981; Altuchov 1969) and was named the "Global Spectrographic Method" (GSM), since the calculations used data from a network of detectors distributed across the globe. Around the same time, Japanese researchers proposed their methodology (Nagashima 1971). In essence, in all variants this is a method of spherical analysis, where the expansion of the function of cosmic ray variations in spherical harmonics is used. But this takes into account cascade processes in the atmosphere and the movement of particles in the Earth's magnetosphere. With this in mind, we can say that GSM is essentially a sophisticated ver-

$\dagger$ deceased 
sion of spherical analysis. IZMIRAN has created its own version of GSM (Belov et al. 1973, 1974, 2019), which has been used for many years for a detailed and comprehensive study of variations in cosmic rays of the zero harmonic (isotropic component of cosmic ray variations), the first spherical harmonic (e.g. Belov et al., 2017), and sometimes the second harmonic of cosmic ray variations.

In the simplest case, the problem is reduced to solving a system of equations describing the cosmic ray variations at each point and obtaining the parameters of the isotropic component variation.

Directly observed variations in the count rate of the $i$-th cosmic ray detector (where $\mathrm{N}_{\mathrm{B}}$ is the base count rate relative to which the variations are calculated) can be represented as

$$
v_{i}=\int_{R_{c}^{i}}^{\infty} W^{i}\left(R, R_{c}^{i}, h_{0}^{i}\right) \cdot \frac{\delta J}{J(R)} \cdot d R+\sigma^{i}
$$

Here $W^{i}\left(R, R_{c}^{i}, h_{0}^{i}\right)$ is a coupling function between primary cosmic ray and variations of the secondary cosmic ray registered by detector $i$ located in the point with geomagnetic cut off rigidity $R_{A}^{i}$ on the atmospheric depth ho. The spectrum of the primary cosmic radiation and the desired spectrum of the isotropic variation are designated, respectively, as $J(R)$ and $\delta J / J(R)$. The discrepancy $\sigma^{i}$ reflects the inadequacy of the used model of variations and possible hardware variations.

The coupling functions determine the influence of the Earth's atmosphere, and the magnetosphere effect takes into account the rigidity of the geomagnetic cutoff of the considered detector. The coupling functions of the neutron component were calculated by us (Aleksanyan et al. 1982), the coupling functions of the muon component were used from Fujimoto et al. (1976).

An important situation is when the analytical form of the desired solution is known, or the solution can be approximated with a high degree of reliability by a model built on the basis of a priori information. In this case, as a rule, the corresponding system of equations for finding a small number of unknown parameters turns out to be well conditioned. The advantage of this situation is that it is practically impossible to obtain an absurd solution if the analytical model is chosen in accordance with the process under study.

The objective of this work is to establish the analytical form of the spectrum of variation with a minimum number of parameters for use in analyzing data from the global network of ground-based cosmic ray detectors using the AMS-02 experimental data on the spectrum of protons and nuclei in the range of rigidity from several GV to several tens of GV. This will make it possible to abandon the empirically specified spectra of variation.

\section{Data of magnetic spectrometer AMS-02}

High-precision data of the orbital detector of cosmic rays AMS-02 for the study of dark matter and antimatter also provide a unique opportunity to measure spectral characteristics at moderate and high rigidity.

Direct measurements of galactic cosmic rays in the range of rigidities from one to several tens of GV were carried out on the PAMELA and AMS-02 spacecraft. The statistical accuracy of the data of the AMS-02 magnetic spectrometer is significantly higher, since the geometric factor of the AMS-02 is about $0.5 \mathrm{~m} 2 \mathrm{sr}$, and the PAMELA spectrometer is only $21.5 \mathrm{~cm} 2 \mathrm{sr}$. This work was based on the data of the AMS-02 magnetic spectrometer.

AMS-02 (Aguilar et al. 2015, 2018) is a magnetic spectrometer operating onboard the International Space Station from 2011 to the present. The digital data is available in the database (CRDB 2020). A description of the database can be found in Valeria et al. (2017).

Sampling by rigidity or by time $(\mathrm{t}, \mathrm{R})$ is possible with fixing one of the parameters. For a given moment in time, proton spectra can be obtained in a very wide range of rigidities. In this work, we limited ourselves to the particle rigidity in the range $1-20 \mathrm{GV}$, to which the network of ground-based neutron monitors is most sensitive. 
The entire time period is divided into 79 intervals averaged by the Carrington rotation since May 2011 (2110 rotations). For example, the time dependence of the proton flux for two energy ranges close to the rigidity of $10 \mathrm{GV}$ can be found in Belov et al. (2020).

\section{Ground methods}

In Belov et al. (1998) a variant of the global spectrographic method is described, specially adapted for studying the long-term behavior of isotropic CR variations. The analysis is carried out using the monthly average data of neutron monitors (about 45 detectors), muon telescopes, and stratospheric sounding. In the simplest case of isotropic variations the problem is reduced to solving the system of equations (1). In the model, it was assumed that the spectrum of variations is given in a threeparameter form and is defined as:

$$
\delta J / J(R)=a_{1} /\left(R_{L}+R\right)^{\gamma}
$$

Area of the parameter changes $\gamma=0 \div 2$ and: $R_{L}=(0 \div 4) \mathrm{GV}$.

A three-parameter approximation of the GCR variation spectrum is suitable for describing the spectrum of long-term variations in the 5-50 GV regions.

Figure 1 shows the parameters of the spectrum of variations in galactic CR: $a_{10}, R_{L}$ and $\gamma$, obtained by this method. The bottom panel shows the standard deviations of the experimental data from the model, which make it possible to assess the adequacy of the applied variation model.

The spectrum of variations in the form (2) was chosen empirically, but other options were also considered. A direct experimental verification of the shape of the spectrum of variations in the range of rigidity from one to several tens of GV has never been carried out, since the maximum rigidity of particles in measurements of fluxes outside the magnetosphere at best reached $2 \mathrm{GV}$. Direct measurements in a wide range of rigidities using unique magnetic spectrometers will solve this problem.

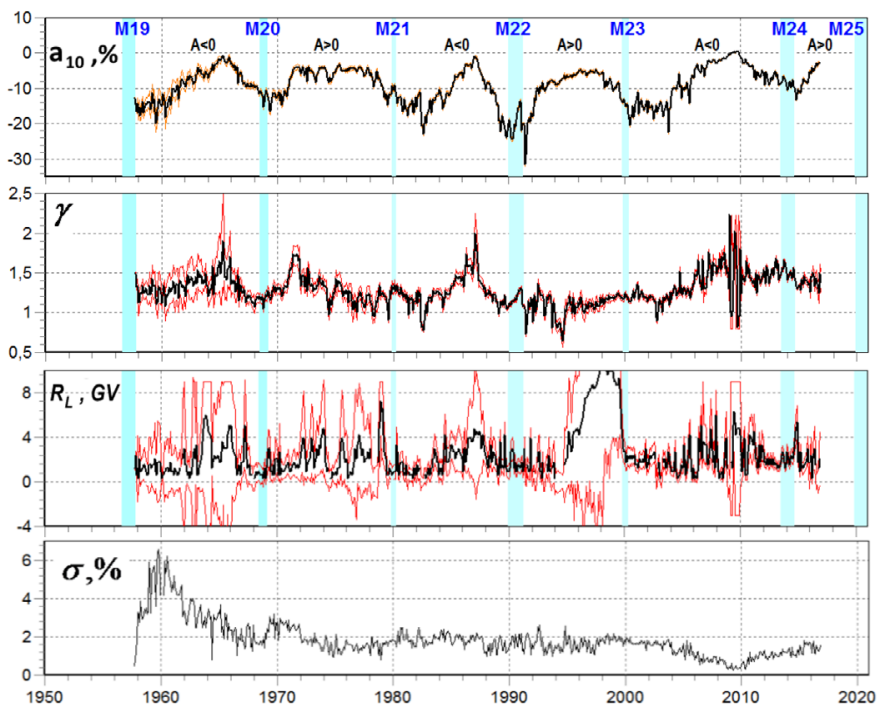

Figure 1: Parameters of the galactic cosmic rays variation spectrum: $a_{10}, R_{L}$ and $\gamma$ the model discrepancy is shown at the bottom. 


\section{Method of experimental determination of the spectrum of variations}

To experimentally determine the spectrum of GCR variations, we used data from the AMS-02 magnetic spectrometer for the entire period of its operation. The time course of AMS-02 flux variations relative to the 2009 base period and their comparison with the GSM results are shown in the top panel of figure 2 . The period of the polarity reversal of the Sun magnetic field from the second half of 2013 to the first half of 2014 is also highlighted here (Sun 2015; Ishkov 2018). But above all, the measured flux was freed from the contribution of solar cosmic rays.

For this, the periods of solar events were excluded from the analysis: 13 SPE, including 6 GLEs (see table 1). The selection criterion for the events was the excess of the proton flux $>100 \mathrm{MeV}$ threshold of $1 \mathrm{pfu}$ (graphical data can be found on the website (SWPC 2020)).

Analysis shows that the contribution $\left(<10^{-4 \%}\right)$ of solar cosmic rays can be neglected by considering the averaged GCR flux per Carrington revolution. In figure 2 (top left panel), the SPE and GLE moments during the AMS-02 operation period are marked with vertical segments, the length of which is proportional to the effect.

\begin{tabular}{|c|c|c|c|}
\hline$d t$ & Flash score & $\begin{array}{c}p>100 \mathrm{MeV}, \\
\text { pfu }\end{array}$ & GLE, \% \\
\hline 2011-08-04 03:41 & M9.3 & 2 & \\
\hline 2011-08-09 07:48 & $x 6.9$ & 2.5 & \\
\hline $2012-01-2303: 38$ & M8.7 & 2.3 & \\
\hline $2012-01-27$ 17:37 & $\mathrm{X} 1.7$ & 11.9 & 7.0 \\
\hline 2012-03-07 00:02 & $\times 5.4$ & 70 & 0.1 \\
\hline 2012-03-13 17:12 & M7.9 & 2 & \\
\hline 2012-05-17 01:25 & M5.1 & 20 & 15.0 \\
\hline $2013-04-1106: 55$ & M6.5 & 2 & \\
\hline $2013-05-22$ 13:08 & M5.0 & 3 & \\
\hline 2014-01-06 07:45 & & 4.1 & 3.0 \\
\hline 2014-01-07 18:04 & $\mathrm{X} 1.2$ & 4.2 & \\
\hline 2015-10-29 02:19 & & 2 & 1.0 \\
\hline $2017-09-10$ 15:35 & $\mathrm{X} 8.2$ & 50 & 6.0 \\
\hline
\end{tabular}

Table 1: Proton enhancements for operating period of the AMS-02.

The result of the analysis in figure 2 is shown for the period of negative polarity (left) and positive polarity (right) of the Sun magnetic field, and the time point is marked with a vertical line in the upper panel of the figure.

The middle panel shows the spectrum of particles (protons) $\mathrm{J}$ for the considered moment of time and the spectrum of particles $J_{B}$ for the base period. Due to the lack of data for a later period, January - April 2017 was selected as the base period (horizontal segment on the top panel), although the SA minimum and the maximum count rate are observed in April 2020. The results should be considered as preliminary and should be revised after updating the AMS-02 data.

Changes in the particle spectrum relative to the particle spectrum in the base period determine the spectrum of variations. By definition, the spectrum of variations for each point in time is defined as:

$$
v=J / J_{B}-1
$$

In the last expression, the variables are functions of time and rigidity, i.e. $v(t, R), J(t, R)$ and $J_{B}(R)$. The experimental spectrum of variations, in this way determined, is shown in the lower panel of figure 2 (points and errors in their determination) for two polarities of the total magnetic field of the Sun. 

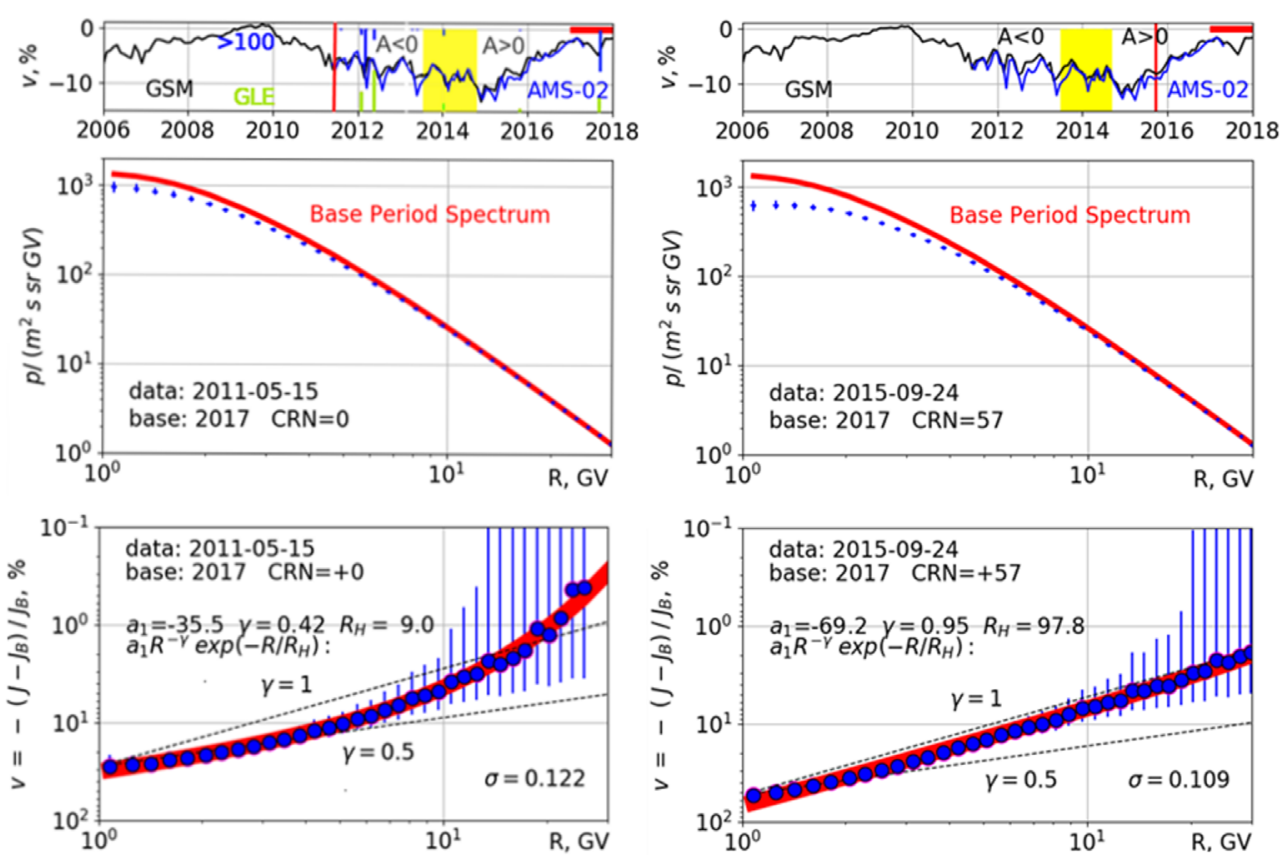

Figure 2: An example of spectra for two points in time: for a period of negative (left) and positive polarity (right) of the total magnetic field of the Sun. The middle panels show the particle spectra (obtained from AMS02) and the spectra of variations found on their basis, shown in the lower panels.

The choice of the approximating function for the spectrum of variation depends on the rigidity range and the problem being solved. Several analytical models of the spectra of cosmic ray variations were considered, including the spectrum of variations of the form (2).

However, the most successful for approximating the experimental spectrum of cosmic ray variations in a wide range of rigidities was the power-law spectrum modulated by an exponential in the region of upper rigidities, i.e.

$$
v=a_{1} R^{-\gamma} \exp \left(-R / R_{H}\right)
$$

where $R_{H}$-upper rigidity. Function (4) is nonlinear with respect to the parameters $a_{1}, \gamma$ and $R_{H}$. System (4) can be reduced to linear by taking the logarithm. As a result, we obtain a system of linear equations for the parameters $\ln a_{1}, \gamma$ and $1 / R_{H}$ :

$$
\ln v_{i}=\ln a_{1}-\gamma \ln \left(R_{i}\right)-R_{i} / R_{H}
$$

The parameters of approximation $a_{1}, \gamma$ and $R_{H}$ for two time moments with positive and negative polarity of the total magnetic field of the Sun also presented at the bottom panels in figure 2 .

More common model:

$$
v=a_{1}\left(R_{L}+R\right)^{-\gamma} \exp \left(-R / R_{H}\right)
$$

In the region of lower rigidities $v$ is also controlled by a parameter, but the model has not yet been considered, since it remains nonlinear even after the logarithm procedure. 


\section{Discussion of the results}

The parameters of approximation $a_{1}, \gamma$, and $R_{H}$ in the considered model (4) were obtained for each solar rotation since May 2011. In the period of negative polarity of the solar magnetic field $(\mathrm{A}<0)$ before the polarity reversal, the parameter $R_{H}$ is close to $10 \mathrm{GV}$, and during this period, a powerlaw spectrum of GCR variations with strong exponential decay in the areas of rigidity $>20 \mathrm{GV}$ is observed. With a change in polarity and at the beginning of a new 24th solar cycle, the parameter $R_{H}$ becomes close to $100 \mathrm{GV}$, and the spectrum turns into a purely power-law spectrum.

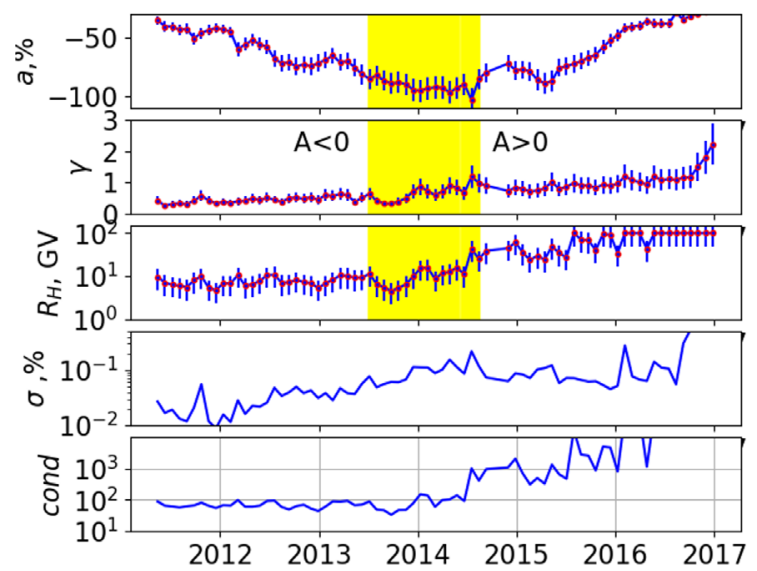

Figure 3: Time dependence of the parameters of the CR spectra variations by the AMS- 02 data.

For the entire period according to AMS-02 data in figure 3 the time dependence of the parameters of the spectrum of variations in cosmic rays are presented. The values of a1, $\gamma$, and $R_{H}$ were obtained as a result of approximation of the experimental spectra, which are shown in figure 2 . The peculiarities of the behavior of the parameters $\gamma$ and $R_{H}$ in periods of different polarities of the solar magnetic field are clearly visible. The $\gamma$ exponent increases linearly over the period under consideration, and the RH parameter increases abruptly from an average value of $\sim 10 \mathrm{GV}$ to $\sim 100 \mathrm{GV}$ after a period of polarity reversal of the solar magnetic field, which is accompanied by a transition to a power-law spectrum of cosmic ray variations. The last two panels in figure 3 show the time dependence of the model error $\sigma$ and the condition number «cond». The value of the conditionality number until the end of the polarity reversal is at the level of 100 , which indicates a well-conditioned system of equations (4). When approaching the base period, the condition number increases rapidly.

It can be noted that before the solar activity maximum in 2014 and the polarity reversal, the experimentally measured variations on the AMS-02 experiment in some periods are almost twice as large as the variations obtained by the GSM method from the data of ground-based detectors. This coincides with the period of negative polarity $\mathrm{A}<0$ of the solar magnetic field, in the period of positive polarity A $>0$ after the polarity reversal of the solar magnetic field, this effect is not observed.

\section{Summary and conclusions}

Due to its unique capabilities, the satellite experiment AMS-02 for the first time made it possible to obtain an experimental spectrum of cosmic ray variations in the range of rigidities to which the ground-based network of neutron monitors is sensitive. This, in turn, will make it possible to remove the uncertainty in the choice of the spectrum of cosmic ray variations during GSM analysis, since until now the spectrum of cosmic ray variations was determined only empirically. 
In this work, based on AMS-02 data, for the first time, the experimental spectrum of cosmic ray variations and its temporal changes in the range of rigidity 1-20 GV for the period from May 2011 to April 2017 are obtained. The found form of the spectrum of variations will make it possible to increase the accuracy of the reconstructed parameters of the spectrum of cosmic ray variations outside the magnetosphere from the data of the network of ground-based cosmic ray detectors.

An important result of this work is the transformation of the form of the spectrum at different periods of polarity of the interplanetary magnetic field. During the period of negative polarity $\mathrm{A}<0$ of the solar magnetic field, a power-law spectrum of variations with a strong exponential decay is observed in the region of upper rigidities.

When the polarity changes $A>0$ the spectrum of cosmic ray variations transforms into a purely power-law spectrum.

In the work for the base period, the data of January - April 2017 were used, but when new data are published, including the minimum SA and the maximum count rate in April 2020, the calculations of the spectrum of variations must be updated.

\section{Acknowledgments}

The work was carried out on the experimental basis USU („,Russian national network of ground cosmic ray stations“). We are grateful to all the staff of the World Network of cosmic ray stations: http://cr0.izmiran.ru/ThankYou

\section{References}

Adriani, 0., Barbarino, G. C., Bazilevskaya, G. A. et al. (PAMELA Collaboration), 2017, "Ten years of PAMELA in space", Riv. Nuovo Cimento 40, 473-522, DOI: https://dx. doi.org/10.1393/ncr/i2017-10140-x; Google Scholar (last accessed April 6, 2021)

Aguilar, M., et al. (AMS Collaboration), 2015, Phys. Rev. Lett. 114, 171103; DOI: https://dx.doi.org/10.1103/PhysRevLett.114.171103

Aguilar, M., et al. (AMS Collaboration), 2018, Phys. Rev. Lett. 121, 051101, DOI: https://dx.doi.org/10.1103/physrevlett.119.251101

Alanko, K., Usoskin, I. G., Mursula, K., Kovaltsov, G. A., 2003, Adv. Space Res., 32 (4), 615-620, DOI: https://dx.doi.org/10.1016/S0273$1177(03) 00348-X$

Belov, A. V., Dorman, L. I. and Yanke, V. G., 1983, "The simplest versions of the global-spectrographical method". Proc. of 18-th ICRC, Bangalore, Vol. 10, 144-147

Belov, A. V., Gushchina, R., T., Yanke, V.,G., 1998, Geomagnetism and Aeronomy, V.38, No.4, 131

Belov, A. V., Gushchina, R.T., Shlyk, N.S., Yank,e V.G.,"Comparison of long-term variations of the cosmic ray flux from the network of ground-based detectors, PAMELA and AMS-02 data", Cosmic ray studies with neutron detectors 1 DOI: https://dx.doi.org/10.38072/2748-3150/p2

CRDB (Cosmic Ray DataBase), 2020, https://tools.ssdc.asi.it/CosmicRays/chargedCosmicRays.jsp (last accessed April 6, 2021)

CR Network, 2020, nmdb: https://www.nmdb.eu/, https://www.nmdb.eu/nest/, usu: http://www.ckp-rf.ru/usu/433536, idb: http://cr0. izmiran.ru/mosc (last accessed April 6, 2021)

DiFelice, V., Pizzolotto, C., D'Urso, D., Dari, S., Navarra, D., Primavera, R., Bertucci, B., 2017, "Looking for cosmic ray data? The ASI Cosmic Ray Database" 35th ICRC, PoS 1073, Korea, https://pos.sissa.it/301/1073/pdf (last accessed April 6, 2021)

Ishkov, V. N., 2018, Geomagnetism and Aeronomy, V.58, No.6, 753-767, DOI: https://dx.doi.org/10.1134/S0016793218060051

Koldobskiy, S. A., Kovaltsov, G. A., Usoskin, I. G., 2018, J. Geophys. Res. 123, 4479, DOI: https://dx.doi.org/10.1029/2018JA025516

Koldobskiy, S. A., Bindi V., Corti C., Kovaltsov G. A., Usoskin I.G., 2019, J. Geophys. Res. 124, 2367-2379. doi: https://dx.doi. org/10.1029/2018JA026340

Koldobskiy, S. A., Bindi, V., Corti, C., Kovaltsov, G. A., Usoskin, I. G., 2019, PoS ICRC 2019, id 1094, https://pos.sissa.it/358/1094/pdf (last accessed April 6, 2021)

Krymskiy, G. F., Altukhov, A. M., Kuzmin, A. I., Krivoshapkin, P. A., Skripin, G. V., Chirkov, N. P., 1966. Geomagnetism and Aeronomy, V.6., No.6, pp.991-996.

Lafferty, G. D., Wyatt, T. R., 1995, "Where to stick your data points: The treatment of measurements within wide bins", Nuclear Instruments and Methods in Physics Research A 355, 541-547, DOI: https://dx.doi.org/10.1016/0168-9002(94)01112-5

Nagashima, K., 1971, "Rep. of lonosphere and Space Res", Japan, Vol. 25, No 3, 189

Sun X., Hoeksema J.T., Liu Y., Zhao J., 2015, Astroph. J., 798, 2, id. 114, DOI: https://dx.doi.org/10.1088/0004-637X/798/2/114

SWPC, 2020, https://www.swpc.noaa.gov (last accessed April 6, 2021)

Ting S., 2013, The Alpha Magnetic Spectrometer on the International Space Station, Nucl. Phys. B, Proc. Suppl. 243-244, 12-24

Usoskin, I. G., Alanko-Huotari, K., Kovaltsov, G. A., and Mursula, K., 2005, J. Geophys. Res., 110, A12108, D0I: https://dx.doi. org/10.1029/2005JA011250 
Usoskin, I. G., Gil, K., Kovaltsov, G. A., Mishev, A. L. and Mikhailov, V. V. , 2017, J. Geophys. Res. Space Physics, 122, 3875-3887, D0I: https://dx.doi.org/10.1002/2016JA023819

Usoskin, I. G., Bazilevskaya, G. A., Kovaltsov, G. A., 2011, Journal of Geophysical Research: Space Physics 116, A02104. D0I: https:// dx.doi.org/10.1029/2010JA016105

Xudong, S., Todd, H. J., Yang, L., Junwei, Z., 2015, Astroph. J., 798, 2, id. 114, 8, DOI: https://dx.doi.org/10.1088/0004-637X/798/2/114

\section{Questions and answers}

Ludwig Klein: Would you argue that the calibration of Pamela after 2012 is not reliable?

Answer: No, I wouldn't, it cannot be asserted. The PAMELA data up to and including 2009 and the AMS-02 data for the entire period are in good agreement with the data of neutron monitors, with the PAMELA data used as the base period of 2009. After a long break since 2010, there were problems with the PAMELA detector, it is evident from the increased statistical errors.

Mike Snow: The $\sim 5 \%$ shift in 2010 PAMELA data is from the PAMELA collaboration or is factor that gives agreement with neutron monitor data?

Answer: A shift of $4.88 \%$ is a factor necessary for agreement with neutron monitor data, but for agreement only since 2010. The PAMELA data up to and including 2009 and the AMS-02 data for the entire period are in good agreement with the data of neutron monitors. The main conclusions of the work are not affected by the normalization of the PAMELA data after 2009. 